does not contain any point of $E_{r}$ other than $p . C_{p}(\eta)$ is defined as in (5). From this it can be shown that $E_{r}$ has finite $(n-1)$-dimensional measure. Let us not assume now that $r$ is large. We then write $E=\bigcup_{k=1}^{m} E^{(k)}$ where the $E^{k}$ s are closed and their diameter is less than $\epsilon$. Then, by what has been shown before, if $\epsilon$ is small enough $E_{r}^{(\boldsymbol{k})}$ has finite $(n-1)$-dimensional measure. Clearly $E_{r} \subset \bigcup_{k=1}^{m} E_{r}^{(k)}$. But $E_{r}$ is closed, therefore its $(n-1)$-dimensional measure exists, and it clearly can not be 0 , since it separates the space. Thus $E_{r}$ must have finite $(n-1)$-dimensional measure.

Added in proof. The author has recently discovered that the following two theorems have been stated by C. Pauc, Revue Scientifique vol. 77 (1939) no. 8: Let the set $E$ be in the plane then $M_{2}$ is contained in the sum of countably many Jordan curves and $M_{3}$ is countable.

UNIVERSITY OF MICHIGAN

\title{
REMARK ON TAYLOR'S FORMULA
}

Taylor's formula

$$
\text { PHILIP HARTMAN }
$$

$$
f(a)=\sum_{k=0}^{n-1} f^{(k)}(0) a^{k} / k !+f^{(n)}(\xi) a^{n} / n !, \quad 0<\xi<a,
$$

is usually proved under the assumptions that

(I) $f(x)$ is continuous on the closed interval $[0, a]$;

(II) $f(x)$ possesses $n-1$ derivatives on the half closed interval $[0, a)$;

(III) $f^{(n-1)}(x)$ is continuous at $x=0$; and

(IV) $f(x)$ has an $n$th derivative on the open interval $(0, a)$.

In the case $n=1$, the assumption (III) that $f^{(0)}(x) \equiv f(x)$ be continuous at $x=0$ is essential but is contained in condition (I). In the case $n>1$, it will be shown below that the assumption (III) is entirely superfluous, so that (1) is valid whenever (I), (II) and (IV) hold.

The proof of (1) is usually reduced to an application of the mean value theorem to the $(n-1)$ th derivative of $f(x)$ on an interval $[0, c]$, $0<c<a$. Thus, to prove the italicized statement, it is sufficient to show that if $f(x)$, defined on the interval $[0, a]$, is the derivative of a function and $f(x)$ itself possesses a derivative on the open interval $(0, a)$, then there exists a number $\xi$ such that

Received by the editors May 18, 1945. 


$$
f(a)-f(0)=a f^{\prime}(\xi) \text { and } 0<\xi<a .
$$

Without loss of generality, it may be supposed that $f(a)=f(0)=0$. It must then be proved that there exists a number $\xi, 0<\xi<a$, such that $f^{\prime}(\xi)=0$. Now if $f(x)$ is identically zero, nothing remains to be proved. Suppose, therefore, that for some $\eta, 0<\eta<a, f(\eta) \neq 0$. Since the function $f(x)$ is a derivative and, consequently, assumes every value between $0=f(0)$ and $f(\eta) \neq 0$ at least once on the interval $(0, \eta)$, there exists a number $x_{1}$, such that

$$
f\left(x_{1}\right)=f(\eta) / 2 \text { and } 0<x_{1}<\eta .
$$

Similarly, there exists a number $x_{2}$ such that

$$
f\left(x_{2}\right)=f(\eta) / 2 \text { and } \eta<x_{2}<a .
$$

Since $f(x)$ possesses a derivative on $(0, a)$, it is continuous on the interval $\left[x_{1}, x_{2}\right]$, and so by the mean value theorem, there exists a number $\xi$ such that

$$
0=f\left(x_{1}\right)-f\left(x_{2}\right)=\left(x_{2}-x_{1}\right) f^{\prime}(\xi) \text { and } x_{1}<\xi<x_{2} .
$$

This completes the proof.

Queens College 